\title{
EFFECT OF PLASTICIZER ON LDPE AND ITS BIODEGRADATION
}

\author{
A. Gadrea , V. Sangawar* and G. Yerawarb \\ *Polymer Research Laboratory, G.V.I.S.H. Amravati (M.S.) INDIA \\ aDepartment of Physics, G.H.R.C.E.M. Amravati (M.S.) INDIA \\ bDepartment of Physics, Arts, Commerce and Science College, Arvi (M.S.)
}

\begin{abstract}
:
With the excess use of plastics products causes very serious problem of accumulation of plastics in environment. An attempt has been made to synthesize degradable plastics. The thin composite films of LDPE + PEG were synthesized by solution evaporation method. The sample was degraded by soil burial technique in compostable soil for 90 days and the samples were characterised by XRD, Fourier Transform IR, and UV - Visual spectroscopy before and after degradation. The degradability is confirmed from crystallinity, carbonyl index and UV - VIS absorption. The LDPE + PEG composite thin films after soil burial of 90 days are found to be degraded. Thus, with the addition of PEG in low density polyethylene (LDPE) makes an environmental friendly and degradable material in natural soil environment.
\end{abstract}

Key words: Biodegradation, LDPE + PEG, Soil burial.

\section{Introduction:}

Plastics are synthetic or semi synthetic, most commonly derived from petrochemicals products by petrochemical industry, but many are partially natural. It refers to their malleability or plasticity during manufacture that allows them to be cast, pressed, or extruded into a variety of shapes — such as films, fibres, plates, tubes, bottles, boxes, small paper pin to large spacecrafts and much more. Polyethylene (PE) is a non-degradable polymer being used extensively in many applications. It is first commodity of plastic to be used for packaging since from 1950. They are used in large scale as packaging material for food and beverages because of low cost and versatile properties. Research is now going towards in replacing some or all kind of synthetic polymers such as $\mathrm{PE}$, to become biodegradable materials due to their pollution in environment. 
The degradation of conventional plastics such as polyethylene, polypropylene, polystyrene, poly (vinyl chloride) and poly (ethylene terphthalate) by the action of natural herbs polymers such as $\operatorname{starch}^{[1]}$, cellulose ${ }^{[2]}$, lignin[10] ${ }^{[10}$ chitin $^{[10]}$ is a significant way to accelerate polymer biodegradation. Microorganism breaks the polymeric chain and consumes materials through aerobic and anaerobic process.

The objective of present study is to investigate the biodegradation of LDPE (low density polyethylene)and PEG(polyethylene glycol)added LDPE composite thin films in compostable soil. (Soil and compost are in 1:1 proportion) The effect of plasticizer, PEG in biodegradation of LDPE thin film have been carried out.

\section{Materials and Methods}

Commercial grade LDPE with density $0.924 \mathrm{~g} / \mathrm{cm}^{3}$ at $23^{\circ} \mathrm{C}$, melt flow index $4 \mathrm{gm} / 10 \mathrm{~min}$; melting range $105-109^{\circ} \mathrm{C}$ were supplied from Nutan Gujarat Industrial Estate, Vadodara (India). Xylene as solvents of AR Grade and PEG with average molecular weights 35004000 , freezing point $53-50{ }^{\circ} \mathrm{C}$ and having $\mathrm{pH}$ in between 4.5 to 7.5 is used as provided without further purification. The compostable soil $(1: 1$ proportion) has $\mathrm{pH} 7.72$.

\section{Sample Preparation}

The thin films of LDPE and LDPE+PEG with 5\% of PEG in LDPE were synthesized by solution evaporation technique ${ }^{[4]}$. Concentration of PEG in LDPE is 5\% and the samples are coded as LP5P0 and LP5P9 for before and after 90 days of degradation respectively. Thickness of the film was measured with high accuracy and resolution using compound microscope in conjunction with an occulometer having 'least count' 15.38 um at magnification of 1:100. A Germal make microscope supplied by Paul Waechter, Model No. 641640 was used [3]. The thickness of the film is in range of 20-25 $\mu \mathrm{m}$. 


\section{Soil Burial Technique}

A soil burial technique is carried out in natural environment to degrade the sample. For this, a thin rectangular sheet of sample (about $2.5 \mathrm{~cm} \times 12 \mathrm{~cm}$ ) of $20-25 \mu \mathrm{m}$ thickness is buried at the depth of $5 \mathrm{~cm}$ in compostable soil having $\mathrm{pH} 7.72$ for 90 days. The moisture content was maintained by adding water in soil after regular interval. The samples were removed from soil after 90 days and washed with distilled water and then acetone bath and dried at room temperature. The sample were characterised by XRD, FTIR and UV absorption spectroscopy.

\section{Result and Discussion:}

\section{X-ray Diffraction (XRD)}

X-ray diffraction of sample LP5P0 and LP5P9 were recorded on XPERT-PRO 11023505 SAIF PU, Chandigarh and are shown in Figures (i) and (ii) respectively.

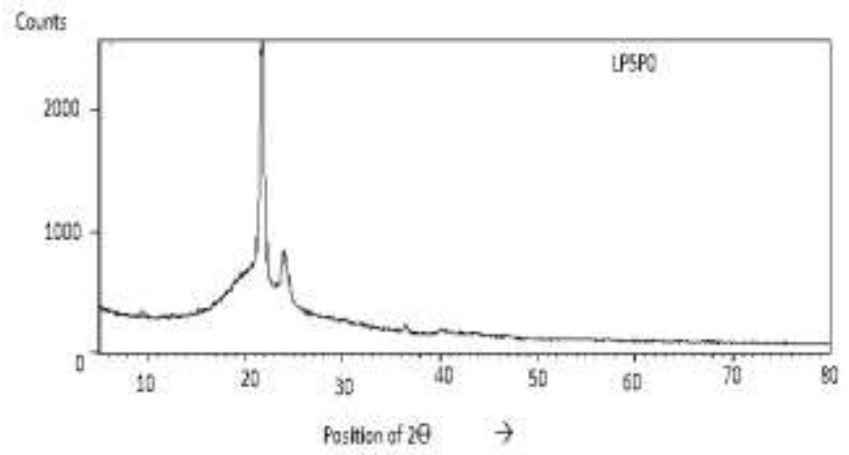

Fig. (i) XRD of LP5PO

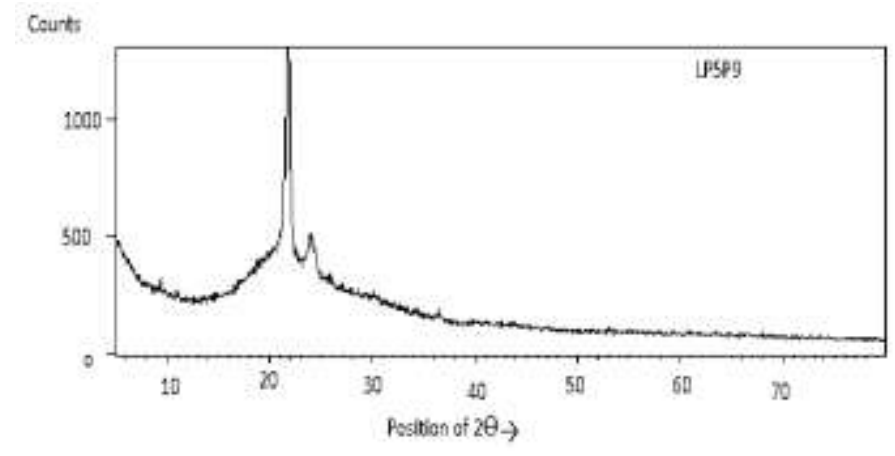

Fig. (ii) XRD of LP5P9

Fig.(i) shows the XRD pattern of thin film of LDPE with 5\% PEG (LP5P0) before crystalline phase along with amorphous phase has been observed in XRD by Peacokok A J[9]. et.al(2000). Diffraction from the (110) and (200) planes in undoped LDPE were centred at $2 \theta=21.9^{\circ}$ and $2 \theta=24.2$ ${ }^{0}$ corresponding to $\mathrm{d}$-spacing of $4.05 \mathrm{~A}^{0}$ and $3.75 \mathrm{~A}^{0}$ respectively $\mathrm{XRD}$ pattern of LP5P0 [Fig.(i)] shows very sharp, small, prominent peaks at 
$2 \theta=21.79^{\circ}$ and $2 \theta=24.04^{\circ}$ corresponding to d-spacing of $4.07 \mathrm{~A}^{0}$ and $3.70 \mathrm{~A}^{0}$ and XRD pattern of LP5P9 [Fig.(ii)] shows very sharp, small, prominent peaks at $2 \theta=21.68^{\circ}$ and $2 \theta=23.910$ corresponding to $\mathrm{d}$ spacing of $4.09 \mathrm{~A}^{0}$ and $3.72 \mathrm{~A}^{0}$. XRD pattern of LP5PO and LP5P9 thin films also suggest small crystalline phase along with amorphous phases but addition of PEG in LDPE matrix changes the crystallinity. The crystallinity was higher for pure LDPE sample but composite of LDPE and PEG changes the crystalline phase and increases amorphous phase. Thin film sample LP5P0 shows decrease in crystalline phase and increase in amorphous phase than undoped and fresh LDPE. The 90 days soil burial sample (LP5P9) shows somewhat more reduction in crystalline phase and more increase in amorphous phase than undoped and fresh LDPE and LP5P0 samples. Thus, it has been found that physically hinder crystal growth resulting in lowering the polymer crystallinity [5] [6].

\section{Fourier Transform Infrared Spectrometer}

FTIR Spectra of thin film samples LP5P0 and LP5P9 was recorded in wavelength region $400-4000 \mathrm{~cm}^{-1}$ FTIR model Perkin Elmer Spectrum RX1, RC SAIF PU, Chandigarh.

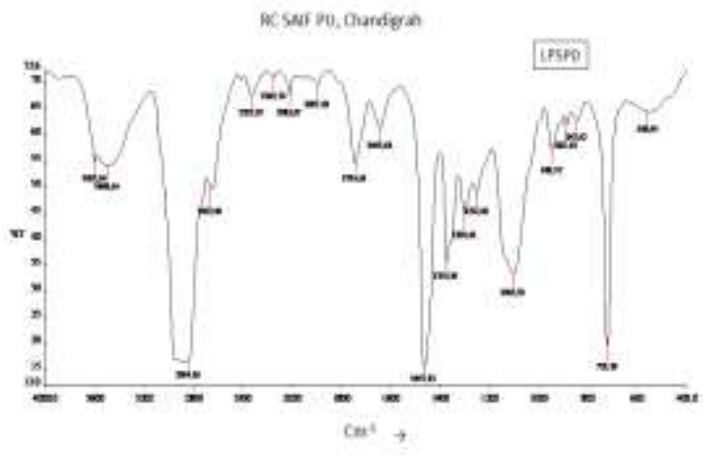

Fig. (iii) FTIR of LP5PO

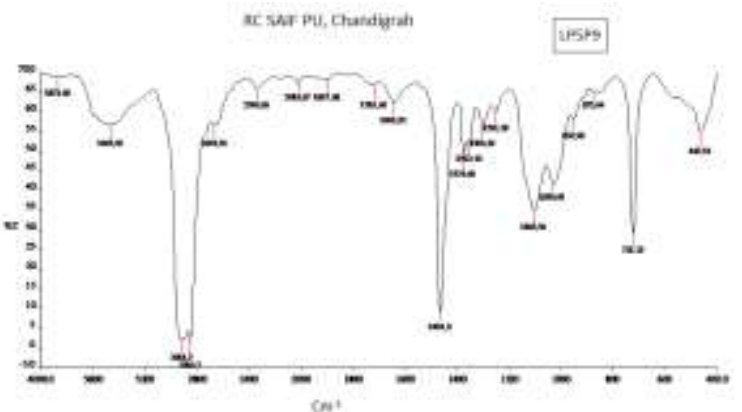

Fig. (iv) FTIR of LP5P9

Figure. (iii) shows the FTIR spectra of LDPE with 5\% PEG (LP5P0) before degradation and Fig. (iv) shows the FTIR spectra of (LP5P9) after 90 days soil burial sample. The spectrum of LP5PO shows peak at $2844.16 \mathrm{~cm}^{-1}$ 
due to asymmetric and symmetric $\mathrm{CH}_{2}$ stretching vibration. The Peak at $1467.15 \mathrm{~cm}^{-1}$ and $721.19 \mathrm{~cm}^{-1}$ corresponds to $\mathrm{C}-\mathrm{H}$ bending and racking amorphous of $\mathrm{CH}_{2}$ group respectively. The band at about $3498.54 \mathrm{~cm}^{-1}$ belongs to $\mathrm{O}-\mathrm{H}$ stretching vibration. The peaks at $1745.54 \mathrm{~cm}^{-1}$ and $1643.61 \mathrm{~cm}^{-1}$ are due to $\mathrm{C}=\mathrm{O}$ stretching of carbonyl group and vinyl group respectively may be due to introduction of carboxylic acid. The spectra of LP5P9 shows peaks at $2914.2 \mathrm{~cm}^{-1}$ and $2854.2 \mathrm{~cm}^{-1}$ are due to asymmetric and symmetric $\mathrm{CH}_{2}$ stretching vibration of LDPE. The Peaks at $1464.9 \mathrm{~cm}^{-1}$ and $721.29 \mathrm{~cm}^{-1}$ correspond to $\mathrm{C}-\mathrm{H}$ bending and racking amorphous of $\mathrm{CH}_{2}$ group respectively. The band at about $3463.56 \mathrm{~cm}^{-1}$ belongs to $\mathrm{O}-\mathrm{H}$ stretching vibration. The peaks at $1719.66 \mathrm{~cm}^{-1}$ and $1641.62 \mathrm{~cm}^{-1}$ are due to $\mathrm{C}=\mathrm{O}$ stretching of carbonyl group and vinyl group may be due to introduction of carboxylic acid. The appearance of these oxidative products on the surface of weathered sample was an indication of oxidative photo degradation [7]. The increase in carbonyl and vinyl group formation for polyethylene after weathering is indication of cross linking and chain scission. [6]

\section{UV-VIS Absorption}

UV-VIS Spectral analysis of sample LP5P0 and LP5P9 has been carried out by using a Hitachi-330 UV-VIS Spectrometer 200-400 nm at SAIF PU, Chandigarh.
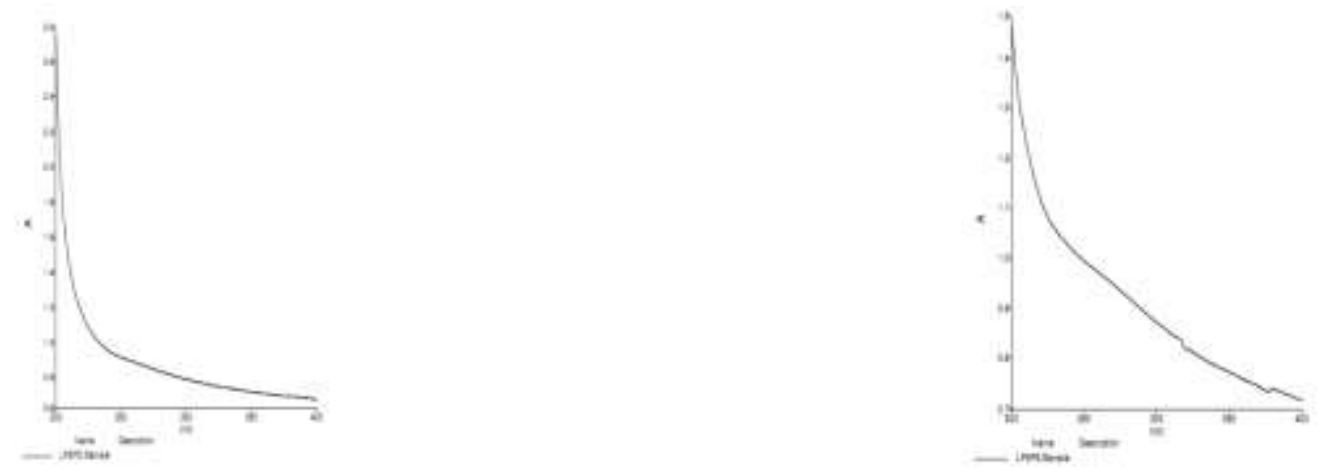

Fig. (v) UV-VIS spectrum of LP5PO

Fig. (vi) UV-VIS spectrum of LP5P9 
Figure (v) shows UV-VIS absorption spectra of LDPE with 5\% PEG(LP5P0) before degradation and Figure (v) shows UV-VIS absorption spectra of (LP5P9) after 90 days soil buried sample. The spectrum of LP5P0 shows very low UV absorption in the region 200-300 $\mathrm{nm}$. The spectra of LP5P9 show some changes in UV absorption in region 200-300 $\mathrm{nm}$. This may be due to presence of few chromophoric groups such as hydro-peroxides, carbonyl and certain catalyst residues which absorb UV radiation in the wavelength range $200-300 \mathrm{~nm}$. [8]

\section{Conclusion}

The XRD results of LP5P0 and LP5P9 shows that the addition of PEG in LDPE affects crystallinity of LDPE that enhances biodegradation of LDPE. FTIR study shows that the appearance of some kind of oxidative product on the surface of weathered sample as an indication of oxidative photo degradation of thin film of LDPE and the increase in carbonyl and vinyl group formation for polyethylene after weathering is the indication of cross linking and chain scission. UV-VIS absorption study indicate that the presence of few chromophoric groups such as hydro-peroxides, carbonyl and certain catalyst residues which absorb UV radiation in the wavelength range $200-300 \mathrm{~nm}$. The XRD, FTIR and UV study of LDPE and LDPE+PEG degraded (for 90 days) sample revealed that addition of PEG affect nature and structure of LDPE and it also helps LDPE in biodegradation. 


\section{References}

Borghei M, Karbassi A,Khoramnejadian S, Oromiehie A and Javid A H , (2010). African Jr. Biotechn. 9(26) 4075-4080.

Behjat T, Russly A R, Luqman C A, Yus A Y and Nor Azowa I - (2009). Inte. Food Research Jr. 16, 243-247.

Sangawar V S, Chikhalikar P S, Dhokane R J, Ubale A U and Meshram S D, (2006). Bull. Mater. Sci. 29(4) 413-416.

Sangawar V S, Meshram S D , Chikhalikar P S ,Dhokane R J, Thool V S and Ubale A U, (2008). J Polym. Mater. Vol. 25 No. 4, 563-575.

Xie X L, Li K Y, Tjong S C, (2002). Polym. Comp. 23, 319-324.

Stark N M and Mastuana L M , (2004). Polym. Degrad. Stab. 86, 19-26.

Mastuana L M and Kamdem D P , (2002). Polym. Eng. Sci. 42, 16571662.

Scoponi M, Cimmino S, Kaci M, (2000). Polymer 41(22) 7969-7980.

Peacock A.J, (2000). Handbook of Polyethylene, Stru. Prop. and Applications, Marcel Dekker, N.Y.

Yutaka Tokiwa, Buenaventurada P, Calabia Charles U, Ugwu and Seiichi Aiba - (2009). Int. J. Mol. Science 10, 3722-3742. 